\title{
Nontrivial RR two-form field strength and SU(3)-structure
}

\author{
Peter Kaste, Ruben Minasian, Michela Petrini and Alessandro Tomasiello \\ Centre de Physique Théorique, Ecole Polytechnique ${ }^{1}$ \\ 91128 Palaiseau Cedex, France \\ kaste, ruben, petrini, tomasiel@cpht.polytechnique.fr
}

\begin{abstract}
We discuss how in the presence of a nontrivial RR two-form field strength and nontrivial dilaton the conditions of preserving supersymmetry on six-dimensional manifolds lead to generalized monopole and Killing spinor equations. We show that the manifold is Kähler in the ten-dimensional string frame if $F_{0}^{(1,1)}=0$. We then determine explicitly the intrinsic torsion of the $\mathrm{SU}(3)$-structure on six-manifolds that result via Kaluza-Klein reduction from seven-manifolds with $\mathrm{G}_{2}$-structure of generic intrinsic torsion. Lastly we give explicitly the intrinsic torsion of the $\mathrm{SU}(3)$-structure for an $\mathcal{N}=1$ supersymmetric background in the presence of nontrivial RR two-form field strength and nontrivial dilaton.
\end{abstract}

\section{Introduction}

A better understanding of $\mathcal{N}=1$ supersymmetric compactifications of string theory to four dimensions is an important step towards more realistic string theories. A promising avenue to take is to break the $\mathcal{N}=2$ supersymmetry of the well-studied Calabi-Yau compactifications of type II string theories down to $\mathcal{N}=1$ by including a background of RR field strength that may describe either internal RR fluxes or spacetime filling D-branes. Of course their presence back-reacts on the metric. In particular the new supersymmetric ground state is no longer a Calabi-Yau manifold. It is an interesting question to study the geometry of these minimally supersymmetric ground states and to characterize how their structure deviates from the one of a Calabi-Yau.

In 1 these questions have been addressed for compactifications of type IIA with a background of nontrivial RR two-form field strength and nontrivial dilaton. This is the situation that is easiest to analyze, since the triple $(g, F, \varphi)$ of the groundstate metric $g$, the background two-form field strength $F$ and the dilaton $\varphi$ can be described in terms of a $\mathrm{G}_{2}$ manifold $Y$. Namely this is the internal manifold of the purely geometrical Mtheory compactification which via Kaluza-Klein reduction gives rise to the above type IIA configuration.

\footnotetext{
${ }^{1}$ Unité mixte du CNRS et de l'EP, UMR 7644
} 
In these proceedings we first construct explicitly an $\mathrm{SU}(3)$-structure $\left(g_{X}, J, \psi_{3}\right)$ on the six-dimensional base space $X$ obtained by Kaluza-Klein reduction from a sevendimensional manifold $Y$ with $\mathrm{G}_{2}$-structure. We then analyze the constraints that preserving $\mathcal{N}=1$ supersymmetry in four dimensions imposes on this $\mathrm{SU}(3)$-structure by requiring the $\mathrm{G}_{2}$-structure on $Y$ to be torsion-free. These constraints can be cast into a primitivity constraint on $F$, a monopole equation relating $d \varphi$ to $F$ and a Killing spinor equation on the associated $\mathrm{SU}(3)$-invariant spinor. It immediately follows that the manifold $\left(X, g_{X}, J\right)$ is Kähler in the string frame if the primitive part of $F^{(1,1)}$ vanishes.

Next we compute explicitly the intrinsic torsion of the $\mathrm{SU}(3)$-structure $\left(g_{X}, J, \psi_{3}\right)$ on $X$ for a generic $\mathrm{G}_{2}$-structure on $Y$. Specializing this result to the case of torsion-free $\mathrm{G}_{2^{-}}$ structure, we give the intrinsic torsion of the $\mathrm{SU}(3)$-structure for an $\mathcal{N}=1$ supersymmetric background in the presence of nontrivial $\mathrm{RR}$ two-form field strength and nontrivial dilaton. Concretely, we show that in the notation of [2] its components are given by $W_{1}=W_{2}^{-}=$ $W_{3}=0, W_{2}^{+}=-\check{F}_{0}^{(1,1)}, W_{4}=-(\beta-2 \alpha) d \varphi$ and $W_{5}=-(\beta-3 \alpha) d \varphi$.

This intrinsic torsion is the obstruction for the Levi-Civita connection of $\left(X, g_{X}\right)$ to have holonomy $\mathrm{SU}(3)$. It can therefore be seen as a measure of how the manifold fails to be a Calabi-Yau. Recently the concept of intrinsic torsion of $G$-structures has been applied to compactifications with background fields in [3, 4, 5, 6, 7]. In [4] it is in particular argued to describe the mirror of NS three-form fluxes in an otherwise purely geometrical mirror compactification. For $\mathcal{N}=1$ supersymmetric compactifications to three dimensions in the presence of background fields $F$ and $\varphi$ and for further references we refer to [1].

\section{From a $\mathrm{G}_{2}$-structure to an $\mathrm{SU}(3)$-structure}

Let $Y$ be the seven-dimensional manifold to which one lifts in M-theory and $g_{Y}$ the background metric on $Y$. Via Kaluza-Klein reduction it is related to the background metric $g_{X}$ on the internal space $X$ of the type IIA compactification by

$$
d s_{Y}^{2}=e^{-2 \alpha \varphi} d s_{X}^{2}+e^{2 \beta \varphi}(d z+A)^{2},
$$

where $A$ is the RR one-form potential and $\varphi$ the dilaton of type IIA. The parameters $\alpha$ and $\beta$ determine the frame of $g_{X}$ in ten-dimensional type IIA. They take the values $(\alpha, \beta)=(1 / 3,2 / 3)$ for the string frame.

Suppose $Y$ carries a $\mathrm{G}_{2}$-structure. The latter is specified by the doublet $\left(g_{Y}, \Phi\right)$ where $\Phi$ is a $\mathrm{G}_{2}$-invariant, nowhere vanishing three-form on $Y$. It can be represented as

$$
\Phi=\frac{1}{3 !} \phi_{A B C} \hat{e}^{A} \hat{e}^{B} \hat{e}^{C},
$$

where $\hat{e}^{A}$ for $A=1, \ldots, 7$ is a frame of orthonormal one-forms w.r.t. $g_{Y}$ and $\phi_{A B C}$ are the structure constants of the imaginary octonions. Moreover, the $\mathrm{G}_{2}$-structure singles out a unique $\mathrm{G}_{2}$-invariant spinor $\epsilon$. It is real (Majorana) and satisfies

$$
\gamma_{A B} \epsilon=i \phi_{A B C} \gamma^{C} \epsilon \quad \text { and } \quad \phi_{A B C}=-i \epsilon^{\dagger} \gamma_{A B C} \epsilon .
$$

If and only if the $\mathrm{G}_{2}$-structure is torsion-free, the Levi-Civita connection associated to $g_{Y}$ has holonomy in $G_{2}$ and in that case the spinor $\epsilon$ will be covariantly constant w.r.t. the Levi-Civita connection. This spinor $\epsilon$ on $Y$ is then the internal part of the supersymmetry 
generator in the $\mathcal{N}=1$ supersymmetric M-theory compactification on $Y$. It is projectible onto $X$ along the $\mathrm{U}(1)$-fibers of the Kaluza-Klein bundle $\pi: Y \mapsto X$ if it is constant along these fibers. In that case it becomes the internal part of the supersymmetry generator in type IIA. Note that since this constant along the fiber may vary over $X$, the spinor $\epsilon$ as seen on $X$ has a U(1) gauge symmetry. In particular $X$ need only carry a $\operatorname{Spin}^{c}$-structure and not a Spin-structure.

If $\epsilon$ is projectible and whether the $\mathrm{G}_{2}$-structure is torsion-free or not, the identity (2.3) projects on $X$ onto

$$
\gamma_{a b} \epsilon=i \psi_{a b c} \gamma^{c} \epsilon+i J_{a b} \gamma \epsilon \quad \text { and } \quad \gamma_{a} \gamma \epsilon=-i J_{a b} \gamma^{b} \epsilon,
$$

where we have defined $\psi_{a b c} \equiv \phi_{a b c}$ and $J_{a b} \equiv \phi_{a b 7}$ for $a, b, c=1, \ldots, 6$ and where $\gamma=\gamma^{7}$ is the chirality operator on $X$. Furthermore

$$
\psi_{a b c}=-i \epsilon^{\dagger} \gamma_{a b c} \epsilon \quad \text { and } \quad J_{a b}=-i \epsilon^{\dagger} \gamma_{a b} \gamma \epsilon .
$$

The spinor $\epsilon$ on $X$ can hence be used to build nowhere vanishing forms

$$
\psi_{3} \equiv \frac{1}{3 !} \psi_{a b c} e^{a} e^{b} e^{c} \quad \text { and } \quad J \equiv \frac{1}{2} J_{a b} e^{a} e^{b}
$$

on $X$. Moreover, since $J_{a}{ }^{b} J_{b}{ }^{c}=-\delta_{a}{ }^{c}$, this $J_{a}{ }^{b}$ defines a natural almost complex structure on $X$ with respect to which $g_{X}$ is automatically hermitian, with associated two-form $J$. Splitting each tangent plane into a holomorphic and antiholomorphic space w.r.t. $J_{a}{ }^{b}$ one sees that $\psi_{3}$ is the real part of a $(3,0)$-form $\Omega=\psi_{3}-i\left(* \psi_{3}\right)$. In particular the forms $\psi_{3}$ and $J$ are not only nowhere vanishing on $X$, they are also by construction invariant under the action of $\mathrm{SU}(3)$ on the tangent bundle $T X$. Altogether, the triple $\left(g_{X}, J, \psi_{3}\right)$ defines an $\mathrm{SU}(3)$-structure on $X$. Its associated $\mathrm{SU}(3)$ invariant spinor is $\epsilon$.

In summary, if $Y$ is a (Kaluza-Klein) $\mathrm{U}(1)$-bundle over $X$ and has a $\mathrm{G}_{2}$-structure with an associated projectible $\mathrm{G}_{2}$-invariant spinor, then the base $X$ carries an $\mathrm{SU}(3)$-structure. This implies that the structure group of $T X$ is $\mathrm{SU}(3)$. However, the $\mathrm{SU}(3)$-structure on $X$ will generically have torsion, even if the $\mathrm{G}_{2}$-structure was torsion-free. I.e. although there exist connections on $T X$ that are compatible with the metric and have holonomy $\mathrm{SU}(3)$, generically none of them will be torsion-free. In that case the Levi-Civita connection cannot have holonomy $\mathrm{SU}(3)$ and $X$ is not a Calabi-Yau. The intrinsic torsion of the $\mathrm{SU}(3)$-structure $\left(X, g_{X}, J, \psi_{3}\right)$ is the obstruction for it to be a Calabi-Yau.

In order to see how $X$ fails to be a Calabi-Yau, we look at the differential equations satisfied by $\epsilon$ on $X$ or alternatively by $\psi_{3}$ and $J$.

\section{The monopole and Killing spinor equations}

Let the $\mathrm{G}_{2}$-structure on $Y$ be torsion-free so that we preserve $\mathcal{N}=1$ supersymmetry in four dimensions. The covariant constancy of $\epsilon$ on $Y$ reduces to the following system on $X$

$$
\begin{aligned}
\left(D_{a}+\frac{i}{2} \alpha\left(\partial_{b} \varphi\right) J_{a}^{b} \gamma\right) \epsilon+i\left(\frac{1}{2} \alpha\left(\partial_{b} \varphi\right) \psi_{a c}^{b}-\frac{1}{4} \check{F}_{a b} J_{c}^{b}\right) \gamma^{c} \epsilon & =0 \\
\left(\frac{1}{4} \check{F}_{a b} J^{a b}\right) \gamma \epsilon+\left(\frac{1}{4} \check{F}_{a b} \psi_{c}^{a b}-\beta\left(\partial_{a} \varphi\right) J_{c}^{a}\right) \gamma^{c} \epsilon & =0
\end{aligned}
$$


where we have defined $\check{F} \equiv e^{(\alpha+\beta) \varphi} F$ and where $D_{a}$ denotes the covariant derivative w.r.t. the Levi-Civita connection on $\left(X, g_{X}\right)$. Since $\gamma^{A} \epsilon$ are linearly independent, the latter of these equations gives

$$
\begin{aligned}
F^{a b} J_{a b} & =0 & & \Leftrightarrow & J\lrcorner F & =0, \\
\beta\left(\partial_{a} \varphi\right) J_{c}^{a} & =\frac{1}{4} \check{F}^{a b} \psi_{a b c} & & \Leftrightarrow & \beta d \varphi & \left.=\frac{1}{2} \check{F}\right\lrcorner\left(* \psi_{3}\right),
\end{aligned}
$$

where $\lrcorner$ denotes the contraction of forms w.r.t. the metric $g_{X}$. On a Kähler manifold the first of these would imply that $F$ is primitive and even though $\left(X, g_{X}, J\right)$ might not be Kähler we will refer to (3.2a as a primitivity constraint. The second equation is a generalized monopole equation. It relates $d \varphi$ to the $(2,0)$ and $(0,2)$ parts of $F$ w.r.t. the almost complex structure. Inserting these into (3.1a) leads to the following Killing spinor equation on $X$ for $\beta=2 \alpha$,

$$
\left(D_{a}+\frac{i}{2} \alpha\left(\partial_{b} \varphi\right) J_{a}^{b} \gamma-\frac{i}{8}\left[\check{F}_{a b} J_{c}^{b}+\check{F}_{c b} J_{a}^{b}\right] \gamma^{c}\right) \epsilon=0
$$

This implies that the only nonvanishing components of the Nijenhuis tensor associated to the almost complex structure $J_{a}^{b}=-i \epsilon^{\dagger} \gamma_{a}^{b} \gamma \epsilon$ are

$$
N_{b c}^{\bar{a}}=\frac{i}{2}\left(\check{F}_{c \bar{d}} \epsilon_{b}^{\bar{d}} \bar{a}-\check{F}_{b \bar{d}} \epsilon_{c}^{\bar{d}} \bar{a}\right) \quad \text { and } \quad N_{\bar{b} \bar{c}}^{a}=-\frac{i}{2}\left(\check{F}_{\bar{c} d} \epsilon_{\bar{b}}^{d^{a}}-\check{F}_{\bar{b} d} \epsilon_{\bar{c}}^{d a}\right),
$$

where we have used the holomorphic/antiholomorphic basis of $T X$ w.r.t. $J_{a}{ }^{b}$. The almost complex structure defined by the spinor $\epsilon$ is therefore integrable if and only if $F^{(1,1)} \equiv 0$. Since $d J=0$ we find that in this case $\left(X, g_{X}, J\right)$ is Kähler for $\beta=2 \alpha$, i.e. defines a torsion-free $\mathrm{U}(3)$-structure. The Killing spinor equation on $X$ then reduces to

$$
\left(D_{a}+\frac{i}{2} \alpha\left(\partial_{b} \varphi\right) J_{a}^{b} \gamma\right) \epsilon=0
$$

Since $\epsilon$ is not covariantly constant w.r.t. the Levi-Civita connection for nontrivial $\varphi, F^{(2,0)}$ and $F^{(0,2)}$, the $\mathrm{SU}(3)$-structure $\left(X, g_{X}, J, \psi_{3}\right)$ however still has torsion.

\section{The general relation between the intrinsic $\mathrm{G}_{2}$-torsion and SU(3)-torsion}

The intrinsic torsion of the $\mathrm{G}_{2}$-structure $\left(g_{Y}, \Phi\right)$ takes values in $\left(\mathfrak{g}_{2}\right)_{\perp} \otimes T^{*} Y$, where $\mathrm{so}(7)=\mathfrak{g}_{2} \oplus\left(\mathfrak{g}_{2}\right)_{\perp}$ and can be decomposed as [2]

$$
X_{1} \in Y \otimes \mathbb{R}, \quad X_{2} \in \Lambda_{14}^{2} T^{*} Y, \quad X_{3} \in \Lambda_{27}^{4} T^{*} Y, \quad X_{4} \in \Lambda_{7} T^{*} Y,
$$

where $\Lambda_{m}^{n} T^{*} Y$ denotes $n$-forms that transform in the representation $\underline{m}$ of $\mathrm{G}_{2}$. In particular these representations imply that

$$
\left.\left.\left.X_{2}\right\lrcorner_{g_{Y}} \Phi=0, \quad \Phi\right\lrcorner_{g_{Y}} X_{3}=0, \quad(* \Phi)\right\lrcorner_{g_{Y}} X_{3}=0,
$$


where $\lrcorner_{g_{Y}}$ denotes the contraction of forms w.r.t. the metric $g_{Y}$. The components (4.1) are determined through $d \Phi$ and $d(* \Phi)$ as [2]

$$
\begin{aligned}
d \Phi & =X_{1}(* \Phi)+X_{4} \wedge \Phi+X_{3}, \\
d(* \Phi) & =\frac{4}{3} X_{4} \wedge(* \Phi)+X_{2} \wedge \Phi .
\end{aligned}
$$

Let us furthermore introduce the notation

$$
X_{j}=Y_{j}+Z_{j} \wedge \hat{e}^{7}, \quad \text { for } j=1, \ldots, 4 .
$$

Then the constraints (4.2) take the form

$$
\begin{aligned}
\left.X_{2}\right\lrcorner_{g_{Y}} \Phi=0 & \Leftrightarrow\left\{\begin{array}{r}
\left.\left.e^{\alpha \varphi} Y_{2}\right\lrcorner_{g_{X}} \psi_{3}+Z_{2}\right\lrcorner_{g_{X}} J=0, \\
J\lrcorner_{g_{X}} Y_{2}=0,
\end{array}\right. \\
\Phi\lrcorner_{g_{Y}} X_{3}=0 & \Leftrightarrow\left\{\begin{array}{r}
\left.\left.e^{\alpha \varphi} \psi_{3}\right\lrcorner_{g_{X}} Y_{3}-J\right\lrcorner_{g_{X}} Z_{3}=0, \\
\left.\psi_{3}\right\lrcorner_{g_{X}} Z_{3}=0,
\end{array}\right. \\
(* \Phi)\lrcorner_{g_{Y}} X_{3}=0 & \left.\left.\Leftrightarrow \frac{1}{2} e^{\alpha \varphi} J^{2}\right\lrcorner_{g_{X}} Y_{3}+\left(* \psi_{3}\right)\right\lrcorner_{g_{X}} Z_{3}=0 .
\end{aligned}
$$

In the following all the contractions will be taken w.r.t. the metric $g_{X}$ and we will drop the label, i.e. $\lrcorner \equiv\lrcorner_{g_{X}}$.

Analogously the intrinsic torsion of the $\mathrm{SU}(3)$-structure $\left(g_{X}, J, \psi_{3}\right)$ takes values in $\mathrm{su}(3)_{\perp} \otimes T^{*} X$, where $\mathrm{so}(6)=\mathrm{su}(3) \oplus \mathrm{su}(3)_{\perp}$. It can be decomposed as

$$
\begin{array}{ll}
W_{1} \in X \otimes \mathbb{C}, & \left.W_{2} \in \Lambda_{8 \oplus 8}^{(1,1)} T^{*} X\right|_{\text {primitive }}, \\
\left.W_{3} \in\left(\Lambda_{6}^{(2,1)} T^{*} X \oplus \Lambda_{\overline{6}}^{(1,2)} T^{*} X\right)\right|_{\text {primitive }}, & W_{4} \in \Lambda_{3}^{(1,0)} T^{*} X \oplus \Lambda_{\overline{3}}^{(0,1)} T^{*} X, \\
W_{5} \in \Lambda_{3}^{(1,0)} T^{*} X \oplus \Lambda_{\overline{3}}^{(0,1)} T^{*} X, &
\end{array}
$$

where $\Lambda_{m}^{\left(n_{1}, n_{2}\right)} T^{*} X$ denotes $\left(n_{1}, n_{2}\right)$-forms that transform in the representation $\underline{m}$ of SU(3). In particular these representations imply that

$$
\left.\left.\left.J\lrcorner W_{2}=0 \quad, \quad J\right\lrcorner W_{3}=0 \quad, \quad \psi_{3}\right\lrcorner W_{3}=0 \quad \text { and } \quad\left(* \psi_{3}\right)\right\lrcorner W_{3}=0 .
$$

We can similarly express the components of its intrinsic torsion through $d J, d \psi_{3}$ and $d\left(* \psi_{3}\right)$ as $[2$

$$
\begin{aligned}
d J & =\frac{3}{2} \operatorname{Im}\left(\overline{W_{1}} \Omega\right)+W_{4} \wedge J+W_{3} \\
& =\frac{3}{2} W_{1}^{-} \psi_{3}-\frac{3}{2} W_{1}^{+}\left(* \psi_{3}\right)+W_{4} \wedge J+W_{3}, \\
d \psi_{3} & =W_{1}^{+} J^{2}+W_{2}^{+} \wedge J+\operatorname{Re}\left(W_{5} \wedge \bar{\Omega}\right) \\
& =W_{1}^{+} J^{2}+W_{2}^{+} \wedge J+W_{5}^{+} \wedge \psi_{3}+W_{5}^{-} \wedge\left(* \psi_{3}\right), \\
d\left(* \psi_{3}\right) & =W_{1}^{-} J^{2}+W_{2}^{-} \wedge J+\operatorname{Im}\left(W_{5} \wedge \bar{\Omega}\right) \\
& =W_{1}^{-} J^{2}+W_{2}^{-} \wedge J+W_{5}^{+} \wedge\left(* \psi_{3}\right)-W_{5}^{-} \wedge \psi_{3}, \\
d \Omega & =W_{1} J^{2}+W_{2} \wedge J+\overline{W_{5}} \wedge \Omega,
\end{aligned}
$$


where we have used the $(3,0)$-form

$$
\Omega=\psi_{3}-i\left(* \psi_{3}\right)
$$

as well as

$$
W_{j}=W_{j}^{+}-i W_{j}^{-}, \text {for } j=1,2,5 .
$$

The minus signs on the imaginary parts result from the fact that compared to [2] we use the opposite orientation, where $J=e^{14}+e^{25}+e^{36}$.

Using

$$
\begin{aligned}
\Phi & =e^{-3 \alpha \varphi} \psi+e^{-2 \alpha \varphi} J \wedge \hat{e}^{7}, \\
(* \Phi) & =e^{-4 \alpha \varphi}(* J)+e^{-3 \alpha \varphi}\left(* \psi_{3}\right) \wedge \hat{e}^{7}=-\frac{1}{2} e^{-4 \alpha \varphi} J^{2}+e^{-3 \alpha \varphi}\left(* \psi_{3}\right) \wedge \hat{e}^{7},
\end{aligned}
$$

equations (4.3a), (4.3b) and (4.8a)-(4.8d), as well as $\check{F}=e^{(\alpha+\beta) \varphi} F$, one derives the following four identities,

$$
\begin{aligned}
& 3 \alpha \psi_{3} \wedge d \varphi+W_{1}^{+} J^{2}+W_{2}^{+} \wedge J+\operatorname{Re}\left(W_{5} \wedge \bar{\Omega}\right)+J \wedge \check{F} \\
& \quad=-\frac{1}{2} e^{\alpha \varphi} X_{1} J^{2}-\psi_{3} \wedge Y_{4}+e^{3 \alpha \varphi} Y_{3} \\
& (\beta-2 \alpha) J \wedge d \varphi+\frac{3}{2} W_{1}^{-} \psi_{3}-\frac{3}{2} W_{1}^{+}\left(* \psi_{3}\right)+W_{4} \wedge J+W_{3} \\
& \quad=e^{-(\alpha+\beta) \varphi} X_{1}\left(* \psi_{3}\right)+e^{-\beta \varphi} J \wedge Y_{4}-e^{-(\alpha+\beta) \varphi} Z_{4} \psi_{3}+e^{-(\beta-2 \alpha) \varphi} Z_{3} \\
& 2 \alpha J^{2} \wedge d \varphi-J^{2} \wedge W_{4}-\left(* \psi_{3}\right) \wedge \check{F} \\
& \quad=-\frac{2}{3} J^{2} \wedge Y_{4}+e^{\alpha \varphi} \psi_{3} \wedge Y_{2} \\
& (\beta-3 \alpha) d \varphi \wedge\left(* \psi_{3}\right)+W_{1}^{-} J^{2}+W_{2}^{-} \wedge J+\operatorname{Im}\left(W_{5} \wedge \bar{\Omega}\right) \\
& \quad=-\frac{4}{3} e^{-\beta \varphi}\left(* \psi_{3}\right) \wedge Y_{4}-\frac{2}{3} e^{-(\alpha+\beta) \varphi} Z_{4} J^{2}+e^{(\alpha-\beta) \varphi} J \wedge Y_{2}+e^{-\beta \varphi} \psi_{3} \wedge Z_{2} .
\end{aligned}
$$

From these equations we can project onto the various $W_{j}$ 's by suitable contractions and express them through the intrinsic torsion $\left(Y_{j}, Z_{j}\right)$ of the $\mathrm{G}_{2}$-structure. To this end it is convenient to decompose forms as

$$
\begin{aligned}
F & =F^{(0)} J+F_{0}^{(1,1)}+F^{(2,0)}+F^{(0,2)} \\
& \left.\left.\left.\left.\left.=\frac{1}{3}(J\lrcorner F\right) J+F_{0}^{(1,1)}+\frac{1}{8}[(F\lrcorner \bar{\Omega})\right\lrcorner \Omega+(F\lrcorner \Omega\right)\right\lrcorner \bar{\Omega}\right] \\
Y_{2} & =Y_{2}^{(0)} J+Y_{2,0}^{(1,1)}+Y_{2}^{(2,0)}+Y_{2}^{(0,2)} \\
& \left.\left.\left.\left.\left.=\frac{1}{3}(J\lrcorner Y_{2}\right) J+Y_{2,0}^{(1,1)}+\frac{1}{8}\left[\left(Y_{2}\right\lrcorner \bar{\Omega}\right)\right\lrcorner \Omega+\left(Y_{2}\right\lrcorner \Omega\right)\right\lrcorner \bar{\Omega}\right] \\
Z_{3} & =Z_{3}^{(0)} \psi_{3}+\tilde{Z}_{3}^{(0)}\left(* \psi_{3}\right)+\left(Z_{3}^{(1,0)}+Z_{3}^{(0,1)}\right) \wedge J+Z_{3,0}^{(2,1)}+Z_{3,0}^{(1,2)} \\
& \left.\left.\left.=\frac{1}{4}\left(\psi_{3}\right\lrcorner Z_{3}\right) \psi_{3}+\frac{1}{4}\left(\left(* \psi_{3}\right)\right\lrcorner Z_{3}\right)\left(* \psi_{3}\right)+\frac{1}{2}(J\lrcorner Z_{3}\right) \wedge J+Z_{3,0}^{(2,1)}+Z_{3,0}^{(1,2)} \\
Y_{3} & =Y_{3}^{(0,1)} \wedge \Omega+Y_{3}^{(1,0)} \wedge \bar{\Omega}+\left(Y_{3}^{(2,0)}+Y_{3}^{(0,2)}\right) \wedge J+Y_{3,0}^{(2,2)}+Y_{3,0}^{(1,1)} \wedge J+Y_{3}^{(0)} J^{2}
\end{aligned}
$$


where subscripts "0" denote primitive forms. Using projectors such as

$$
\left.\left.\left.\left.\left.\left.Y_{3,0}^{(1,1)}=J\right\lrcorner Y_{3}-\frac{1}{3}\left(J^{2}\right\lrcorner Y_{3}\right) J-\frac{i}{8}(\Omega\lrcorner Y_{3}\right)\right\lrcorner \bar{\Omega}+\frac{i}{8}(\bar{\Omega}\lrcorner Y_{3}\right)\right\lrcorner \Omega,
$$

the components $W_{j}$ of the intrinsic torsion of the $\mathrm{SU}(3)$-structure $\left(g_{X}, J, \psi_{3}\right)$ can be expressed through the components $\left(Y_{j}, Z_{j}\right)$ of the intrinsic torsion of the $\mathrm{G}_{2}$-structure $\left(g_{Y}, \Phi\right)$ as,

$$
\begin{aligned}
W_{1}^{+} & =-\frac{2}{3} e^{-(\alpha+\beta) \varphi} X_{1}-\frac{2}{3} e^{-(\beta-2 \alpha) \varphi} \tilde{Z}_{3}^{(0)} \\
W_{1}^{+}+\check{F}^{(0)} & =-\frac{1}{2} e^{\alpha \varphi} X_{1}+e^{3 \alpha \varphi} Y_{3}^{(0)} \\
W_{1}^{-} & =-\frac{2}{3} e^{-(\alpha+\beta) \varphi} Z_{4} \\
W_{2}^{+}+\check{F}_{0}^{(1,1)} & =e^{3 \alpha \varphi} Y_{3,0}^{(1,1)} \\
W_{2}^{-} & =e^{(\alpha-\beta) \varphi} Y_{2,0}^{(1,1)} \\
W_{3} & =e^{-(\beta-2 \alpha) \varphi}\left[Z_{3,0}^{(2,1)}+Z_{3,0}^{(1,2)}\right] \\
W_{4}+(\beta-2 \alpha) d \varphi & =e^{-\beta \varphi} Y_{4}+e^{-(\beta-2 \alpha) \varphi}\left[Z_{3}^{(1,0)}+Z_{3}^{(0,1)}\right] \\
\left.W_{4}-2 \alpha d \varphi+\frac{1}{2} \check{F}\right\lrcorner\left(* \psi_{3}\right) & \left.=\frac{2}{3} Y_{4}-\frac{1}{2} e^{\alpha \varphi} Y_{2}\right\lrcorner \psi_{3} \\
\left.W_{5}^{(1,0)}-3 \alpha d \varphi^{(1,0)}+\frac{i}{4} \check{F}\right\lrcorner \Omega & \left.=Y_{4}^{(1,0)}-\frac{1}{4} e^{3 \alpha \varphi} \Omega\right\lrcorner Y_{3} \\
W_{5}^{(1,0)}+(\beta-3 \alpha) d \varphi^{(1,0)} & \left.=\frac{4}{3} e^{-\beta \varphi} Y_{4}^{(1,0)}-\frac{1}{4} e^{(\alpha-\beta) \varphi} Y_{2}\right\lrcorner \Omega-i e^{-\beta \varphi} Z_{2}^{(1,0)}
\end{aligned}
$$

The decomposition of the $X_{j}$ 's into the $W_{j}$ 's at the level of representations figures already in 2]. Equations (4.15a)-(4.15j) determine the explicit coefficients that appear in this decomposition for a metric (2.1) of the form that appears in Kaluza-Klein reductions to an arbitrary frame.

Let's now specialize again to the case of torsion-free $\mathrm{G}_{2}$-structure, where all the right hand sides vanish. From (4.15a) and (4.15b) we recover the primitivity constraint (3.2a)

$$
\left.\frac{1}{3} J\right\lrcorner F \equiv F^{(0)}=0
$$

whereas equations (4.15g), (4.15h) as well as (4.15i), (4.15j) and their complex conjugates give us the monopole equation (3.2b)

$$
\left.\beta d \varphi=\frac{1}{2} \check{F}\right\lrcorner\left(* \psi_{3}\right) .
$$

In addition to these two constraints, the components of the intrinsic torsion of the $\mathrm{SU}(3)$ structure in the case of torsion-free $\mathrm{G}_{2}$-structure are given by

$$
\begin{array}{lll}
W_{1}^{ \pm}=0 \quad, & W_{2}^{-}=0 \quad, & W_{3}=0, \\
W_{2}^{+}=-\check{F}_{0}^{(1,1)} & W_{4}=-(\beta-2 \alpha) d \varphi \quad, & W_{5}=-(\beta-3 \alpha) d \varphi .
\end{array}
$$


Moreover, we recover the result from the previous section that for $\beta=2 \alpha$ the manifold $\left(X, g_{X}, J\right)$ is Kähler if $F_{0}^{(1,1)}=0$, since then the only nonvanishing component of the intrinsic torsion of $\left(g_{X}, J, \psi_{3}\right)$ is $W_{5}$.

\section{Acknowledgement}

We would like to thank B. Acharya, D. Calderbank, M. Douglas, D. Martelli and A. Moroianu for useful discussions. PK would furthermore like to thank the organizers of the 35th Ahrenshoop Symposium for the invitation to participate in a stimulating conference and the opportunity to present this work. This work is supported in part by EU contract HPRN-CT-2000-00122 and by INTAS contracts 55-1-590 and 00-0334. PK and MP are supported by European Commission Marie Curie Postdoctoral Fellowships under contract numbers HPMF-CT-2000-00919 and HPMF-CT-2001-01277.

\section{References}

[1] P. Kaste, R. Minasian, M. Petrini and A. Tomasiello, "Kaluza-Klein bundles and manifolds of exceptional holonomy," JHEP 0209 (2002) 033, arXiv:hep-th/0206213.

[2] S. Chiossi, S. Salamon, "The intrinsic torsion of $S U(3)$ and $G_{2}$ structures" Proc. conf. Differential Geometry Valencia 2001, math.DG/0202282.

[3] J.P. Gauntlett, D. Martelli, S. Pakis and D. Waldram, "G-structures and wrapped NS5-branes," arXiv:hep-th/0205050.

[4] S. Gurrieri, J. Louis, A. Micu and D. Waldram, "Mirror symmetry in generalized Calabi-Yau compactifications," arXiv:hep-th/0211102.

[5] G.L. Cardoso, G. Curio, G. Dall'Agata, D. Lüst, P. Manousselis, G. Zoupanos, "NonKähler string backgrounds and their five torsion classes," arXiv:hep-th/0211118.

[6] J.P. Gauntlett and S. Pakis, "The geometry of $d=11$ Killing spinors," arXiv:hep-th/0212008.

[7] E. Goldstein and S. Prokushkin, "Geometric model for complex non-Kähler manifolds with SU(3)-structure," arXiv:hep-th/0212307.

[8] M. Cvetic, G. W. Gibbons, H. Lu and C. N. Pope, "Almost special holonomy in type IIA and M theory," Nucl. Phys. B 638 (2002) 186, arXiv:hep-th/0203060. 